\title{
A proof of the (strengthened) Liar formula in a semantical extension of Peano Arithmetic
}

\section{JEFFREY KETLAND}

Let $P A$ be standard first-order Peano Arithmetic in $L$, the first-order language of arithmetic. Let $P A(S)$ be a semantical extension of $P A$ obtained by adding a primitive satisfaction predicate $\operatorname{Sat}_{L}(x, y)$, governed by Tarskian axioms. Let $L_{S}$ be the resulting language-i.e., $L$ plus the new primitive $\operatorname{Sat}_{L}(x, y)$. The system of axioms governing $\operatorname{Sat}_{L}(x, y)$ was given in Tarski 1936. Anyone interested in delving into some highly technical work on this topic could consult Feferman 1991, Kaye 1991 and Halbach 1999.1

The philosophical significance of such semantical extensions is discussed in Ketland 1999, where such extensions are compared with minimalistic extensions generated by adding just the 'T-scheme' (the set of formulas $\operatorname{Tr}_{L}(\ulcorner\varphi\urcorner) \leftrightarrow \varphi$, where $\varphi$ does not contain $\left.\operatorname{Tr}_{L}(x)\right)$. These latter extensions are conservative. Similar constructions are considered in Shapiro 1998. And, as both Shapiro and I argued, the issue of (non-)conservativeness of adding a theory of truth to a base theory can be related to the issue of deflationism about truth. ${ }^{2}$

1 The notation $P A(S)$ is due to Kaye 1991. It is worth mentioning that an ambiguity arises concerning what is meant, in defining the satisfaction-theoretic extension, by 'adding' the new axioms which contain new vocabulary. The ambiguity is whether to expand the induction scheme in $P A$ to include formulas containing $\operatorname{Sat}_{L}(x, y)$. It turns out that if induction is not expanded (which is arguably 'unnatural'), then $P A+$ satisfaction axioms is a conservative extension. However, if induction is expanded, so inductive proofs involving the formulas containing $S a t_{L}$ and $\operatorname{Tr}_{L}$ can be formalized, then the extension $P A+$ satisfaction axioms is a non-conservative extension, and indeed, this is the theory we refer to as $P A(S)$. The non-conservativeness is witnessed by the fact that $P A(S) \vdash \operatorname{Con}(P A)$.

(In fact, the system $P A(S)$ is fully intertranslatable with a certain subsystem of second-order arithmetic called ACA ('Arithmetic Comprehension Axiom'). See Halbach 1999).

2 The crux of the matter is that it can be shown that $P A(S) \vdash \operatorname{True}(P A)$ where True $(P A)$ is the 'soundness' formula $\forall x(\operatorname{Prov}(x) \rightarrow \operatorname{Tr}(x))$ (or 'Global Reflection Principle') and where $\operatorname{Prov}(x)$ is the standard provability predicate for $P A$. So, $P A(S)$ proves that 'any theorem of $P A$ is true'. Indeed, an analogous construction can be given for any sufficiently rich formalized system $F$ (such as $Z F C$ ) resulting in a semantical extension 


\section{JEFFREY KETLAND}

Introduce within this theory the truth predicate $T r_{L}(x)$, governed by the explicit definition,

(1) $\quad \forall x\left(\operatorname{Tr}_{L}(x) \leftrightarrow\left(\operatorname{Sen}_{L}(x) \wedge \forall y\left(\operatorname{Seq}(y) \rightarrow \operatorname{Sat}_{L}(x, y)\right)\right)\right)$

The idea is that $\operatorname{Sat}_{L}(x, y)$ expresses the satisfaction relation between (codes of) $L$-formulas and (codes of) sequences. ${ }^{3}$ Then $\operatorname{Tr}_{L}(x)$ expresses the concept of truth for such $L$ sentences. It can be shown that this theory satisfies Tarski's Convention T: i.e.,

(2) $\quad P A(S) \vdash \operatorname{Tr}_{L}(\ulcorner\varphi\urcorner) \leftrightarrow \varphi$

for each closed formula $\varphi$ of $L$. This syntactic looking fact has a nice model-theoretic corollary. Let $(M, S, T r)$ be any model of $P A(S)$. Let \# $\varphi$ be the gödel number of the $L$-formula $\varphi$. Then the fact (2) entails that, for any closed $L$-formula $\varphi$,

(3) $\# \varphi \in \operatorname{Tr} \Leftrightarrow M \vDash \varphi$

Furthermore, it can easily be shown that any model of $P A(S)$ will assign to $\operatorname{Tr}_{L}(x)$ exactly the (codes of) $L$-sentences that hold in the reduct $M$. That is, it gets the extension of $\operatorname{Tr}_{L}(x)$ exactly right. It is important to notice the presence of the formula $\operatorname{Sen}_{L}(x)$ in the definition of $\operatorname{Tr}_{L}(x)$. It is this which ensures that only (the codes of) $L$-sentences enter extension of $\operatorname{Tr}_{L}(x)$. Hence,

(4) If $(M, S, T r) \vDash P A(S)$ then $\operatorname{Tr}=\{\# \varphi: M \vDash \varphi$ and $\varphi \in \operatorname{Sen}(L)\}$

To return to the main point, there is an $L$-formula $\operatorname{Sen}_{L}(x)$ which strongly defines within $P A(S)$ the (recursive) set of codes of closed $L$-formulas. That is,

(5) If $\varphi$ is a closed $L$-formula, then $\mathrm{PA}(S) \vdash \operatorname{Sen}_{L}(\ulcorner\varphi\urcorner)$

(6) If $\varphi$ is not a closed $L$-formula then $\operatorname{PA}(S) \vdash \neg \operatorname{Sen}_{L}(\ulcorner\varphi\urcorner)$

Furthermore, the definition (1) of $\operatorname{Tr}_{L}(x)$ guarantees that the following holds:

(7) $\quad P A(S) \vdash \forall x\left(\operatorname{Tr}_{L}(x) \rightarrow \operatorname{Sen}_{L}(x)\right)$

$F(S)$. The analogous result is that $F(S) \vdash$ dash $\operatorname{True}(F)$. It is clear that this is a proper extension of $F$, because $F(S) \vdash \operatorname{Con}(F)$. I do not know whether the strength of such systems have been examined in any more detail.

Incidentally, it is known that $P A(S) \vdash P H$, the Paris-Harrington formula which is famously not a theorem of $P A$. See Kaye 1991.

${ }^{3}$ Normally, the metatheory requires a certain amount of set theory. However, only finite sequences are needed to recursively define satisfaction for first-order languages, all of whose formulas contain only finitely many variables. The reason is that the class of finitesequencesofnaturalnumbersiscountableandrecursive:thuseachsuchsequence can be coded (in an effective manner) as a number. Then a predicate $\operatorname{Seq}(x)$ strongly defining this set can be defined in $P A$. Thus, we assume that the sole new concept introduced into $P A(S)$ is the concept expressed by satisfaction predicate $\operatorname{Sat}_{L}(x, y)$. 
Next, think about the Diagonalization Lemma (or Fixed Point Theorem). The system $P A(S)$ satisfies the requirements of this theorem (it is an extension of Robinson Arithmetic $Q$ ). Thus, there must be a fixed point formula $\lambda$ such that,

(8) $\quad P A(S) \vdash \lambda \leftrightarrow \neg \operatorname{Tr}_{L}(\ulcorner\lambda\urcorner)$

The analysis of the proof of the Diagonalization Lemma ${ }^{4}$ shows that this formula $\lambda$ must contain the new predicate $\operatorname{Tr}_{L}(x)$. Indeed, because the truth-in- $L$ predicate $\operatorname{Tr}_{L}(x)$ cannot, by Tarski's Indefinability Theorem, be defined in $P A$, it follows that such a formula is not a closed $L$-formula (and is not logically equivalent to or logically interdeducible with any $L$ formula). Thus, $\lambda$ must be an $L_{S}$ formula.

This formula $\lambda$ is the formal analogue of the so-called 'strengthened liar' for our formalized semantical system $P A(S)$. It is a formula that 'says of itself that it is not true'. But what does 'true' mean here? Well, it has to mean 'true-in- $L$ '. Intuitively, this claim is, in fact, correct: i.e., $\lambda$ is, in fact, not true-in- $L$. Thus, $\lambda$ is, in fact, true (in $L_{S}$ ). This is, in effect, Tarski's own resolution of the liar paradox (including the strengthened liar).

We shall use the above facts $(5)-(8)$ to deduce something rather interesting. Namely, that the strengthened liar formula $\lambda$ is a theorem of $P A(S)$. Thus, $\lambda$ is provably true-in- $L_{S}$ ! The required proof that the formula $\lambda$ is a theorem of $P A(S)$ is triviality itself. Since, as we noted, $\lambda$ is not a closed $L$ formula, we can deduce from (6) that,

(9) $\quad \vdash P A(S) \vdash \neg \operatorname{Sen}_{L}(\ulcorner\lambda\urcorner)$

It immediately follows from (7) that,

(10) $P A(S) \vdash \neg \operatorname{Tr}_{L}(\ulcorner\lambda\urcorner)$

and thus, from (8),

(11) $P A \vdash \lambda$

This interesting result deserves further comment. Intuitively, $P A(S)$ is in fact true (it is certainly true in the standard expansion $(\Re, S, T r)^{5}$ of the

${ }^{4}$ See, e.g., (Boolos Jeffrey 1989). If $\varphi$ is any formula, then the diagonalization of $\varphi$, call it $\operatorname{Diag}(\varphi)$, is the formula $\exists x(x=\ulcorner\varphi\urcorner \wedge \varphi)$. (Note that if $\varphi$ contains the variable $x$ free, then $\operatorname{Diag}(\varphi)$ is equivalent to the formula $\varphi(\ulcorner\varphi\urcorner)$ which obviously says that $\varphi$ is true of the code of itself). Taking codes, we get the diagonal function diag on numbers. This function is provably recursive. So, suppose that the $L$-formula $\operatorname{Diag}(x, y)$ represents (in our theory $P A(S)$ ) this diagonal function diag. Next let $\Phi$ be the $L_{S}$-formula $\exists y\left(\operatorname{Diag}(x, y) \wedge \neg \operatorname{Tr}_{L}(y)\right)$. Finally, let $\lambda$ be $\operatorname{Diag}(\Phi)$. It then quickly follows that $\lambda \leftrightarrow$ $\neg \operatorname{Tr}_{L}(\ulcorner\lambda\urcorner)$ is a theorem of $P A(S)$.

${ }^{5} S$ is the satisfaction relation on the standard model $\Re$. I.e., it is the set of pairs $(n, m)$ where $n$ is the code of an $L$-formula $\varphi$ and $m$ is the code of some finite sequence $s$ and $\mathfrak{N} \vDash_{s} \varphi$; and $\operatorname{Tr}$ is the corresponding set of codes of true formulas (in $\left.\mathfrak{N}\right)$. 
intended structure $\mathfrak{X}$ ). It follows that $\lambda$ is, in fact, true (or, if you like, it holds in $(\Re, S, T r))$. Furthermore, it follows that $\neg \operatorname{Tr}_{L}(\ulcorner\lambda\urcorner)$ is also true! This correctly expresses the fact that the $L_{S}$-formula $\lambda$ is in fact not true in $L$ : it is not true in $L$ for the rather trivial 'syntactic' reason that $\lambda$ is not equivalent to any $L$-formula. Indeed, it turns out that the $\lambda$ 's code - the number $\# \lambda$ - cannot be an element of $T r$, for this set is constrained by the definition of $\operatorname{Tr}_{L}(x)$ given above ((1)) to be a subset of the set (of codes) of closed $L$-formulas. The formula $\lambda$ is the formal equivalent for our formal system $P A(S)$ of the strengthened liar, which allegedly 'says of itself that it is not a true sentence'. But, in our study, this claim is actually true. The strengthened liar sentence $\lambda$ is not even a sentence of $L$, and is a fortiori not a true sentence of $L$. However, $\lambda$ is, in fact, a true sentence of the extended language $L_{S}$ and is, in fact, provable in $P A(S)$.

The London School of Economics Houghton Street, LONDON WC2A $2 A E$ j.j.ketland@lse.ac.uk

\section{References}

Boolos, G. and R. Jeffrey. 1989. Computability and Logic. Third edition. Cambridge: Cambridge University Press.

Feferman, S. 1991. Reflecting on incompleteness. Journal of Symbolic Logic 56: 1-49. Halbach, V. 1999. Conservative theories of classical truth. Studia Logica 62: 353-70.

Kaye, R. 1991. Models of Peano Arithmetic. (Oxford Logic Guides 15). Oxford: Clarendon Press.

Ketland, J. 1999. Deflationism and Tarski's paradise. Mind 108: 69-94.

Shapiro, S. 1998. Truth and proof - through thick and thin. Journal of Philosophy 95: 493-522.

Tarski, A. 1936. Der wahrheitsbegriff in den formalisierten Sprachen. Studia Philosophica 1: 261-405. English translation, by J. H. Woodger, 'The concept of truth in formalized languages', appeared in A. Tarski 1956, Logic, Semantics and Metamathematics: Papers by Alfred Tarski from 1923 to 1938. Oxford: Clarendon Press. 